\title{
Short communication: Field evaluation of a pregnancy confirmation test using milk samples in dairy cows
}

\author{
Stephen J. LeBlanc ${ }^{1}$ \\ Population Medicine, University of Guelph, Canada N1G 2W1
}

\section{ABSTRACT}

The objective was to validate the performance under field conditions of a novel commercially available ELISA for confirmation of pregnancy using measurement of pregnancy-associated glycoproteins in milk samples from dairy cows. The target population was cows previously diagnosed pregnant by veterinary examination and $\geq 60 \mathrm{~d}$ of gestation. On 8 farms milking Holstein cows, milk samples were collected during routine Dairy Herd Improvement testing and shipped overnight to the Dairy Herd Improvement laboratory where the milk pregnancy test was performed. On the same day that milk samples were collected, transrectal palpation was performed by a veterinarian to confirm pregnancy status. Data were available from 683 cows, of which 661 were pregnant and 22 were not pregnant based on veterinary diagnosis, which was taken as the reference test. Based on the manufacturer's interpretive guidelines, $3.8 \%$ of test results were classified as "recheck," between the cut-points for classification of pregnant and nonpregnant and were not used in the analysis. The milk pregnancy test performance (and 95\% confidence intervals) for confirmation of pregnancy was sensitivity of $99.2 \%$ (98.2 to $99.7 \%$ ) and specificity of $95.5 \%$ (78.2 to $99.2 \%$ ). Given a prevalence of $97 \%$ pregnant cows in the sample, the positive predictive value of the milk test was $99.8 \%$ (99.1 to $99.96 \%)$ and the negative predictive value was $80.8 \%$ (61.3 to $90.9 \%$ ). When used to confirm pregnancy status or detect fetal losses at $\geq 60$ $\mathrm{d}$ gestation in cows previously diagnosed pregnant, the recommended action for cows with a milk pregnancyassociated glycoprotein test result of not pregnant is veterinary reexamination of the animal to confirm the presence or absence of a viable fetus before reinsemination or administration of prostaglandin.

Key words: pregnancy diagnosis, pregnancy confirmation, pregnancy-associated glycoprotein

Received November 25, 2012.

Accepted December 21, 2012.

${ }^{1}$ Corresponding author: sleblanc@uoguelph.ca

\section{Short Communication}

Timely pregnancy is economically important for dairy herds and, consequently, timely and accurate pregnancy diagnosis is a key element of management of reproduction. Initial diagnosis of nonpregnancy is necessary so that cows can be reinseminated if not pregnant to a previous AI. Conversely, among cows correctly diagnosed pregnant, loss of pregnancy is common, especially between early diagnosis of pregnancy at 28 to $30 \mathrm{~d}$ of gestation and 56 to $60 \mathrm{~d}$ of gestation, during which time approximately $15 \%$ of pregnancies may be lost (Santos et al., 2004), although these losses are more likely in the earlier part of this interval. From approximately $60 \mathrm{~d}$ of gestation to term, the rate of fetal loss is reduced but 2 to $4 \%$ of pregnancies are typically lost (Santos et al., 2004). Recent data from DHI records in the United States indicate 1.3\% abortion from $151 \mathrm{~d}$ of gestation to term (Norman et al., 2012).

Pregnancy-associated glycoproteins (PAG) are a group of $>20$ proteins produced by binucleate cells in the bovine placenta, with increases detectable in plasma in pregnant cows starting at approximately $24 \mathrm{~d}$ of gestation, and are diagnostically useful at 28 to $30 \mathrm{~d}$ after AI (Romano and Larson, 2010; Thompson et al., 2010; Fricke and Giordano, 2011). The concentrations of various PAG vary with the stage of gestation and decline when fetal loss occurs (Giordano et al., 2012). Recently, a test has been developed to measure PAG in milk, in addition to existing validated tests for PAG in plasma or serum. The objective was to validate the performance of a novel commercially available ELISA for confirmation of pregnancy using milk samples from dairy cows.

The study population was a convenience sample of 8 dairy herds milking Holstein cows in southwestern Ontario, Canada. The herds were chosen based on using DHI service (CanWest DHI, Guelph, ON, Canada), having accurate computerized reproduction records, exclusively using AI, and willingness to participate when asked. The herds were also selected to provide a variety of brands and types of milking equipment (7 freestall barns with parlors and 1 tiestall barn) and milk meters. The herds had between 97 and 496 cows 
being milked (mean \pm SD: $266 \pm 168$ ), with milk production between 33 and $42 \mathrm{~kg} / \mathrm{cow}$ per day $(37 \pm 11$ $\mathrm{kg} /$ cow per day) on the day of sampling. Each herd was visited once in May or June 2012. On the day of the visit, a routine DHI milk-recording test was conducted. A composite milk sample was collected from each cow, preserved with bronopol, and shipped overnight to the DHI laboratory (CanWest DHI). The order of milking (i.e., the sequence of cows milked at each stall in the parlor or with each milking unit) was recorded. At the laboratory, samples from cows $\geq 60$ DIM and $\geq 60 \mathrm{~d}$ pregnant, or not pregnant but $\geq 60 \mathrm{~d}$ since the last AI based on the herd's records on the day of sampling were analyzed using a commercially available 96 -well microplate ELISA for detection of PAG in milk (Idexx Laboratories Inc., Westbrook, ME). Technicians collecting samples and performing the ELISA were blinded to the cows' pregnancy status. The ELISA test was performed by a trained technician according to manufacturer's instructions. A microtiter plate was coated with antiPAG antibody. After incubation in the well, captured PAG was detected with a PAG-specific antibody and a horseradish peroxidase conjugate. Unbound conjugate was washed away, and colorimetric substrate was added to the wells. Color development was proportional to the amount of PAG in the sample and was measured using a spectrophotometer. Results were calculated from the optical density (OD) of the sample (S) minus the OD of the negative control $(\mathrm{N})$ at $450 \mathrm{~nm}$ (with both values corrected by subtraction of the reference wavelength OD), which resulted in an $\mathrm{S}-\mathrm{N}$ value. Each microplate included negative and positive control and reference samples. Based on the manufacturer's guidelines, samples with $\mathrm{S}-\mathrm{N}<0.100$ were classified as not pregnant, $\mathrm{S}-\mathrm{N} \geq 0.100$ but $<0.250$ as "recheck," and $\mathrm{S}-\mathrm{N} \geq 0.250$ as pregnant. Because residual PAG from the previous pregnancy is detectable after calving, the manufacturer's specification is to use the test only in cows $>60$ DIM. Based on the objective of the present study and the intended application of the test, assuming submission of samples through routine monthly DHI testing, samples from cows previously diagnosed pregnant and $\geq 60 \mathrm{~d}$ of gestation were analyzed. Additional samples were taken from cows expected to be nonpregnant (i.e., "do not breed" cows that the farm managers had elected not to inseminate but that were still in the herd lactating) and that were also $\geq 60 \mathrm{~d}$ since the last insemination; these were included to increase the precision of the estimate of the specificity of the test. On 7 farms, essentially all cows meeting the study-inclusion criteria were examined by an experienced veterinarian to confirm pregnancy status by transrectal palpation $[<2 \%$ of cows per farm that met the inclusion criteria were not examined if the cow could not be positively identified (e.g., missing ear tags) or could not be caught]. On one farm, milking 464 cows, not all eligible cows could be physically processed sufficiently quickly through the handling facility (management rail), so a random sample of 219 cows meeting the inclusion criteria was used. Pregnancy was confirmed if placentomes or the fetus was palpated. Veterinarians were aware of the cow's recorded pregnancy status and stage (days of gestation).

The sample size was estimated based on establishment of noninferiority (one-sided test, $99.5 \%$ statistical confidence) of the milk test to within 2 to $2.5 \%$ points of veterinary diagnosis (assuming 2\% discordant results between the milk test and palpation), resulting in sample sizes of 794 or 516 cows, respectively (Abramson, 2011).

Veterinary diagnosis by rectal palpation was taken as the reference test (gold standard) to which the milk pregnancy test was compared. Performance of the latter was assessed by calculating the sensitivity (proportion of pregnant cows classified as pregnant), specificity (proportion of nonpregnant cows classified nonpregnant), positive predictive value (proportion of milk pregnancy test results of "pregnant" for which the cow was pregnant) and negative predictive value (proportion of milk pregnancy test results of "open" for which the cow was not pregnant). These measures and their 95\% confidence intervals (Zou's method) were calculated using WinPepi version 11.22 (Abramson, 2011; http://www.brixtonhealth.com/pepi4windows. html).

Milk pregnancy test results and concurrent veterinary pregnancy diagnosis were available from 710 cows that met the study inclusion criteria, of which $27(3.8 \%)$ had a result of "recheck," leaving 683 cows in the final analysis. Of these, 665 had been previously diagnosed pregnant and 18 were recorded in the farm records as not pregnant; all were $\geq 60 \mathrm{~d}$ since the last AI. Among 661 cows actually diagnosed pregnant on the day of sampling and examination, the mean $( \pm \mathrm{SD})$ stage of gestation was $140 \pm 49 \mathrm{~d}$ (range from 60 to $230 \mathrm{~d}$ ) and among 22 nonpregnant cows, the mean interval from the last AI was $153 \pm 83 \mathrm{~d}$ (range from 61 to $341 \mathrm{~d}$ ). The data and test performance are summarized in Table 1. Sensitivity (classification of pregnant cows as pregnant) was $99.2 \%$ and specificity (classification of open cows as open) was $95.5 \%$. The kappa statistic was 0.87 , indicating overall excellent agreement beyond chance between the diagnostic methods. With 661 of 683 cows pregnant, the positive predictive value (probability that a cow with a milk result of "pregnant" was pregnant) was $99.8 \%$ and the negative predictive value (probability that a cow with a milk result of "open" was open) was $80.8 \%$. 
Table 1. Summary of results of a novel commercially available pregnancy test based on measurement of pregnancy-associated glycoprotein in milk in cows $\geq 60 \mathrm{~d}$ from the last insemination, using veterinary diagnosis of pregnancy by transrectal palpation of the fetus or placentomes as the gold standard ${ }^{1}$

\begin{tabular}{lccc} 
& \multicolumn{2}{c}{ Pregnancy status (veterinarian) } & \\
\cline { 2 - 3 } $\begin{array}{l}\text { Milk test } \\
\text { result }^{2}\end{array}$ & Pregnant & Open & Total \\
\hline Pregnant & 656 & 1 & 657 \\
Open & 5 & 21 & 26 \\
Total & 661 & 22 & 683 \\
\hline
\end{tabular}

${ }^{1}$ For each cow, veterinary examination and collection of the milk sample by routine DHI testing were performed on the same day in Holstein cows from 8 herds in Canada. Test performance (95\% CI): sensitivity $=99.2 \%$ (98.2 to $99.7 \%$ ); specificity $=95.5 \%$ ( 78.2 to $99.2 \%$ ); positive predictive value $=99.8 \%(99.1$ to $99.96 \%) ;$ negative predictive value $=$ $80.8 \%$ (61.3 to $90.9 \%$ ); kappa statistic $=0.87$ (0.77 to 0.97$)$.

2"Recheck" results were not included; $\mathrm{n}=27 / 710$ (3.8\%).

The milk pregnancy test had very high sensitivity for confirmation of pregnancy and, given the target population of cows expected to be pregnant ( $97 \%$ prevalence of pregnant cows in the sample), the correspondingly high positive predictive value implies that users could have high confidence that a milk test result of "pregnant" is accurate. It is important to note that the objective and design of the study was to assess the milk pregnancy test for confirmation of pregnancy at $\geq 60 \mathrm{~d}$ of gestation in cows previously diagnosed pregnant by the herd veterinarian. If samples were taken from a different population in which the prevalence of pregnant cows were lower (e.g., cows not previously diagnosed pregnant) and if the sensitivity and specificity did not change, the positive predictive value would be lower than in the present results.

The negative predictive value (NPV) of $81 \%$ implies that approximately 1 in 5 milk test results of "open" was incorrect. This estimate has a wide confidence interval because of the small number of nonpregnant cows in the study's target population. In the present data, 4 of 665 cows $(0.6 \%)$ expected to be pregnant were not. If only cows expected to be pregnant were tested, given the specificity of $95.5 \%$, the NPV would be lower, simply because so few nonpregnant cows would be in the sample. Conversely, inclusion of more than $4 \%$ nonpregnant cows in the sample would increase the NPV. The actual prevalence of nonpregnant cows depends on the accuracy of the initial pregnancy diagnosis, the rate of subsequent pregnancy loss, and the interval from initial diagnosis of pregnancy to the confirmation test. The recommended course of action for cows previously diagnosed pregnant (at $<60 \mathrm{~d}$ of gestation) but subsequently classified as not pregnant by this milk test is to examine the cow for the presence of a viable fetus by examination by a veterinarian and (or) repeated blood or milk PAG tests. Given the target population for pregnancy confirmation and the performance of the milk pregnancy test, it is not recommended that cows classified as "open" be injected with $\mathrm{PGF}_{2 \alpha}$ or reinseminated without further confirmation of their pregnancy status. Therefore, the utility of the present milk pregnancy test for confirmation of pregnancy may lie in the convenience of confirmation of pregnancy for most pregnant cows without handling the animals.

Data from the 6 cows $(6 / 683=0.9 \%)$ in which the milk pregnancy test and veterinary diagnosis were discordant were examined in detail. These 6 cows were from 4 different herds and no apparent cow or production factors explained these discrepancies. No pattern among these cows was observed with respect to parity (1 to 5 ), stage of lactation (186 to 294 DIM), interval since last AI (113 to $204 \mathrm{~d}$ ), sample day milk production (22 to $59 \mathrm{~kg} / \mathrm{cow} / \mathrm{d}$ ), components (2.9 to $4.6 \%$ fat and 2.8 to $4.0 \%$ protein), or SCC (20 to $368 \times 10^{3}$ cells $/ \mathrm{mL}$ ) One instance occurred of a cow that was not pregnant classified by the milk test as pregnant. In this case, the milk test $\mathrm{S}-\mathrm{N}$ was 0.28 , just above the cut-point of 0.25 for classification as pregnant. The cow milked in the same stall in the parlor immediately before this cow was pregnant, with a milk $\mathrm{S}-\mathrm{N}$ of 2.44. Therefore, one hypothesis to explain the incorrect milk test result is sample carryover (i.e., residual milk in the meter from the previous cow). However, as this was the only instance of this possible problem that was identified among over 700 samples, if this was a case of carryover this would be a rare occurrence.

Five instances occurred of cows that were pregnant but classified as open by the milk pregnancy test. As described above, all of these were from cows at $\geq 113$ $\mathrm{d}$ of gestation at sampling. All of these cows were reexamined by the herd veterinarian 2 to 3 mo after initial sampling. In 3 cases, the cows were confirmed again to be pregnant; the milk PAG $\mathrm{S}-\mathrm{N}$ results were $0.04,0.04$, and 0.06 [i.e., well below the cut-point for pregnancy $(\geq 0.250)$ or recheck $(<0.100)]$; no clear explanation exists for these results. In 2 other cases, at the follow-up veterinary examination, the cows were found to have aborted; in each case, the estimated time of the abortion was 5 to 6 wk after milk sampling and initial pregnancy confirmation. These cows were from different farms, were 113 and 133 d post-AI, and each had a milk $\mathrm{S}-\mathrm{N}$ of 0 at initial sampling. Therefore, it is possible that the milk pregnancy test was correctly indicating a nonviable pregnancy. Experimentally induced pregnancy loss was associated with initiation of decline of plasma PAG concentration within $1 \mathrm{~d}$, but the circulating concentration of PAG may remain above the threshold for classification as pregnant for 7 to 10 
d or more (Giordano et al., 2012). Given the interval from sampling to detection of pregnancy loss, these results remained classified as discordant in the analysis. Little published information exists on the accuracy of transrectal palpation for detection of fetal nonviability or loss $>60 \mathrm{~d}$ after AI and following a previous diagnosis of pregnancy, but the method is very widely used and, therefore, is relevant as the reference test in this analysis.

Because late embryonic and fetal pregnancy losses are common and economically important, confirmation of pregnancy after $60 \mathrm{~d}$ of gestation is recommended. The milk pregnancy test for confirmation of pregnancy assessed in the present field study demonstrated high positive predictive value and moderately high negative predictive value using milk samples that are routinely collected approximately monthly on many dairy farms.

\section{ACKNOWLEDGMENTS}

This study was funded by Idexx Laboratories Inc. (Westbrook, ME) and CanWest DHI (Guelph, ON, Canada). Valuable assistance in sample collection was provided by Christine Gutscher, Julie Wittrock, and Peter Spruit (University of Guelph). The time and interest of participating producers and veterinarians is greatly appreciated.

\section{REFERENCES}

Abramson, J. H. 2011. WINPEPI updated: Computer programs for epidemiologists, and their teaching potential. Epidemiol. Perspect. Innov. 8:1.

Fricke, P. M., and J. O. Giordano. 2011. Use of chemical tests for pregnancy diagnosis in a reproductive management program. Pages 48-56 in Proc. Dairy Cattle Reprod. Conf., Kansas City, MO. Dairy Cattle Reproduction Council, Hartland, WI.

Giordano, J. O., J. N. Guenther, G. Lopes Jr., and P. M. Fricke. 2012 Changes in serum pregnancy-associated glycoprotein, pregnancyspecific protein $\mathrm{B}$, and progesterone concentrations before and after induction of pregnancy loss in lactating dairy cows. J. Dairy Sci. 95:683-697.

Norman, H. D., R. H. Miller, J. R. Wright, J. L. Hutchison, and K. M. Olson. 2012. Factors associated with frequency of abortions recorded through Dairy Herd Improvement test plans. J. Dairy Sci. 95:4074-4084

Romano, J. E., and J. E. Larson. 2010. Accuracy of pregnancy specific protein-B test for early pregnancy diagnosis in dairy cattle. Theriogenology 74:932-939.

Santos, J. E. P., W. W. Thatcher, R. C. Chebel, R. L. A. Cerri, and K. N. Galvão. 2004. The effect of embryonic death rates in cattle on the efficacy of estrus synchronization programs. Anim. Reprod. Sci. 82-83:513-535.

Thompson, I. M., R. L. A. Cerri, I. H. Kim, J. A. Green, J. E. P. Santos, and W. W. Thatcher. 2010. Effects of resynchronization programs on pregnancy per artificial insemination, progesterone, and pregnancy-associated glycoproteins in plasma of lactating dairy cows. J. Dairy Sci. 93:4006-4018. 